\title{
Convergence of semantics and emotional expression within the IFG pars orbitalis
}

Citation for published version (APA):

Belyk, M., Brown, S., Lim, J., \& Kotz, S. A. (2017). Convergence of semantics and emotional expression within the IFG pars orbitalis. Neuroimage, 156, 240-248. https://doi.org/10.1016/j.neuroimage.2017.04.020

Document status and date:

Published: 01/08/2017

DOI:

10.1016/j.neuroimage.2017.04.020

Document Version:

Publisher's PDF, also known as Version of record

Document license:

Taverne

Please check the document version of this publication:

- A submitted manuscript is the version of the article upon submission and before peer-review. There can be important differences between the submitted version and the official published version of record.

People interested in the research are advised to contact the author for the final version of the publication, or visit the DOI to the publisher's website.

- The final author version and the galley proof are versions of the publication after peer review.

- The final published version features the final layout of the paper including the volume, issue and page numbers.

Link to publication

\footnotetext{
General rights rights.

- You may freely distribute the URL identifying the publication in the public portal. please follow below link for the End User Agreement:

www.umlib.nl/taverne-license

Take down policy

If you believe that this document breaches copyright please contact us at:

repository@maastrichtuniversity.nl

providing details and we will investigate your claim.
}

Copyright and moral rights for the publications made accessible in the public portal are retained by the authors and/or other copyright owners and it is a condition of accessing publications that users recognise and abide by the legal requirements associated with these

- Users may download and print one copy of any publication from the public portal for the purpose of private study or research.

- You may not further distribute the material or use it for any profit-making activity or commercial gain

If the publication is distributed under the terms of Article $25 \mathrm{fa}$ of the Dutch Copyright Act, indicated by the "Taverne" license above, 


\title{
Convergence of semantics and emotional expression within the IFG pars orbitalis
}

\author{
Michel Belyk ${ }^{\mathrm{a}, \mathrm{b}, *}$, Steven Brown ${ }^{\mathrm{b}}$, Jessica Lim ${ }^{\mathrm{b}}$, Sonja A. Kotz ${ }^{\mathrm{a}, \mathrm{c}}$ \\ a Faculty of Psychology and Neuroscience, University of Maastricht, Maastricht, The Netherlands \\ b Department of Psychology, Neuroscience \& Behaviour, McMaster University, Hamilton, Ontario, Canada \\ ${ }^{\mathrm{c}}$ Department of Neuropsychology, Max Planck Institute for Human and Cognitive Sciences, Leipzig, Germany
}

\section{A R T I C L E I N F O}

\section{Keywords:}

Inferior frontal gyrus

Pars orbitalis

Emotion

Semantics

Meta-analysis

Parcellation

\begin{abstract}
A B S T R A C T
Humans communicate through a combination of linguistic and emotional channels, including propositional speech, writing, sign language, music, but also prosodic, facial, and gestural expression. These channels can be interpreted separately or they can be integrated to multimodally convey complex meanings. Neural models of the perception of semantics and emotion include nodes for both functions in the inferior frontal gyrus pars orbitalis (IFGorb). However, it is not known whether this convergence involves a common functional zone or instead specialized subregions that process semantics and emotion separately. To address this, we performed Kernel Density Estimation meta-analyses of published neuroimaging studies of the perception of semantics or emotion that reported activation in the IFGorb. The results demonstrated that the IFGorb contains two zones with distinct functional profiles. A lateral zone, situated immediately ventral to Broca's area, was implicated in both semantics and emotion. Another zone, deep within the ventral frontal operculum, was engaged almost exclusively by studies of emotion. Follow-up analysis using Meta-Analytic Connectivity Modeling demonstrated that both zones were frequently co-activated with a common network of sensory, motor, and limbic structures, although the lateral zone had a greater association with prefrontal cortical areas involved in executive function. The status of the lateral IFGorb as a point of convergence between the networks for processing semantic and emotional content across modalities of communication is intriguing since this structure is preserved across primates with limited semantic abilities. Hence, the IFGorb may have initially evolved to support the comprehension of emotional signals, being later co-opted to support semantic communication in humans by forming new connections with brain regions that formed the human semantic network.
\end{abstract}

\section{Introduction}

Humans communicate meaning using a combination of language based on arbitrary acoustic or visual symbols - and emotional expression, including prosodic modulation of speech, but also interjections, facial expressions, and body gestures (Dietrich et al., 2006; Filippi, 2016; Scherer and Ellgring, 2007). While humans use language to provide each other with information, emotional communication is a phylogenetically-older channel that may be used to manipulate the behavior of others (Owren and Rendall, 2001). Some neurological models of speech perception have argued for decades that language and emotional expression are mediated by distinct brain areas in opposite hemispheres (Ross and Mesulam, 1979; Ross and Monnot, 2008). Beyond the criticisms that such models have faced on empirical grounds (Belyk and Brown, 2014; Fusar-Poli et al., 2009b; Witteman et al., 2012, 2014), they raise the important question of how language and emotional expression become integrated during communication. An alternative view is that there might be a convergence of these two facets of communication in particular brain areas, leading to the hypothesis that such areas might serve as hubs to integrate semantics and emotion during communication.

The pars orbitalis division of the inferior frontal gyrus (IFGorb) is one candidate for such an integration zone between semantic and emotional communication. The core semantic network includes the angular gyrus, middle temporal gyrus, posterior cingulate gyrus, dorsomedial prefrontal cortex, and the inferior frontal gyrus (Binder et al., 2009; Binder and Desai, 2011). The core network for perceiving emotional expressions includes the posterior superior temporal cortex, amygdala, anterior insula, basal ganglia, dorsolateral prefrontal cortex, and the inferior frontal gyrus (Belyk and Brown, 2016; Brück et al., 2011; Frühholz et al., 2016). In both of these networks, the inferior frontal gyrus, particularly the IFGorb, has a role in evaluating and

\footnotetext{
* Corresponding author at: Faculty of Psychology and Neuroscience, Maastricht University, Universiteitssingel 40, Maastricht, 6200 MD, The Netherlands.

E-mail address: belykm@gmail.com (M. Belyk).
} 


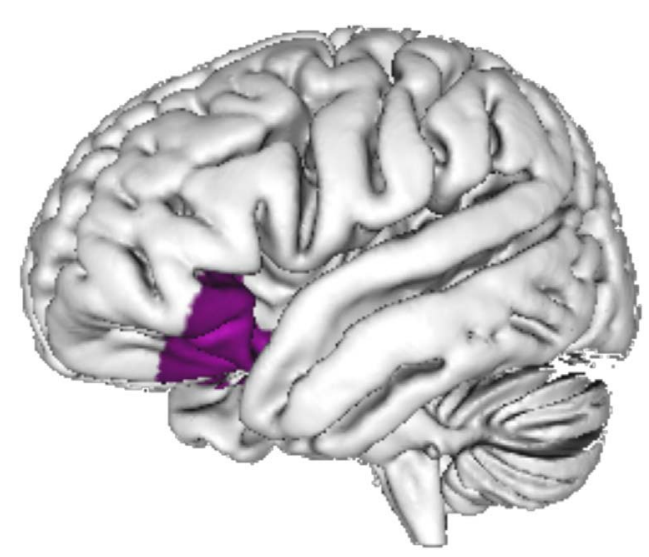

Fig. 1. Location of the IFGorb (purple) in the left hemisphere from the Harvard-Oxford probabilistic cortical map (Craddock et al., 2012).

acting upon communicative signals.

Brain imaging studies in humans have demonstrated that the IFGorb participates in perceiving both semantic content and emotional expression across sensory modalities. For example, this region is activated when participants make judgments about the semantic content of speech, written text, and sign language (Binder et al., 2009; MacSweeney et al., 2002; Rodd et al., 2015), as well as emotional expression through the voice, face, body, and music (Belyk and Brown, 2014; Frühholz et al., 2016; Fusar-Poli et al., 2009a; Jessen and Kotz, 2015; Lehne et al., 2014; Levitin and Menon, 2003; Lotze et al., 2006; Tabei, 2015; Witteman et al., 2012). This leads to the hypothesis that the IFGorb may serve as a hub that integrates semantics and emotion during communication.

The IFGorb is bounded between the orbital sulcus and the anterior horizontal ramus of the lateral sulcus, extending into the frontal operculum. This anatomical region corresponds approximately to the cytoarchitectonic designation of Brodmann area (BA) 47 (Brodmann, 1909) schematized in Fig. 1. Although Brodmann designated this region with a single cytoarchitectonic label, he noted, based on the research of Oskar Vogt, that much of the inferior frontal cortex was myeloarchitectonically diverse (Vogt, 1910; see translation by Judaš and Cepanec, 2010) and thus amenable to further subdivision. Vogt's coarsest-level division separated the opercular IFGorb from the rest of the inferior frontal gyrus, a division that is now corroborated by magnetic resonance imaging parcellation studies in both humans and monkeys (Neubert, Mars, Thomas, Sallet, and Rushworth, 2014; Neubert, Mars, Sallet, and Rushworth, 2015). This leads to a second hypothesis, that possible anatomical subdivisions within the IFGorb may have corresponding functional specializations, such as the comprehension of semantic and emotional signals.

We report two voxel-based meta-analyses of published brain imaging studies that test the hypotheses that the IFGorb contains a zone of convergence between the semantic and emotional-expression networks and/or that it contains multiple functionally-specialized subregions. These hypotheses have implications for the study of both semantic and emotional-expression processing, as research from both fields has independently attributed certain functions to the IFGorb. The first meta-analysis used Kernel Density Estimation (KDE) to test whether published studies reporting loci of activation within the IFGorb during the perception of semantic meaning and emotional expression, respectively, do so in a common zone - which would be consistent with a role in integrating these channels - and/or in spatially distinct zones, which would be consistent with a further subdivision of this region. A second analysis examined networks of coactivation for functionally defined zones within the IFGorb to further inform the interpretation of their functions.

\section{Meta-Analysis 1}

\section{Methods}

Database searching

We searched the BrainMap database (www.brainmap.org) for brain imaging experiments using Sleuth software v2.3 on September $16^{\text {th }}$ 2014 for all brain imaging experiments that reported loci of activation within the cytoarchitectonically defined Brodmann area 47. This literature searching strategy is appropriate for testing where within BA 47 these functions may be localized, but not whether BA 47 is engaged in these functions. It would be circular to support the latter inference from this corpus of studies, although previous meta-analyses (reviewed above) provide ample support for the role of the IFGorb in these functions.

Coordinates for activation loci labeled as either IFG, IFG pars orbitalis, or BA 47 were extracted from the coordinate tables of each study. Coordinates reported in MNI space were transformed to Talairach space. Each coordinate was checked against the Talairach atlas (Talairach and Tournoux, 1988), as implemented in a digital atlas (Lancaster et al., 1997, 2000), to verify that it fell within the cytoarchitectonically defined IFGorb. Loci falling outside of this region were excluded from the analysis. Restricting analyses to this space improves statistical power for testing a priori hypotheses at the expense of conducting lower-powered exploratory analyses across the remainder of the brain volume.

In light of theories of lateralization of linguistic and emotional communication, we performed Chi-squared tests on the number of activation loci and the number of experiments that reported at least one locus of activation in each hemisphere. Studies of semantic perception were strongly left-lateralized (total loci: $\chi^{2}=25.2, p<0.05$; experiments: $\chi^{2}=9, p<0.05$ ), whereas studies of emotion perception showed no evidence of lateralization (total loci: $\chi^{2}=0.049, \mathrm{p}=0.86$; experiments: $\chi^{2}=0, p=1$ ). We therefore restricted our analysis to the left hemisphere, where both functions could be compared.

\section{Study coding}

Each study was manually classified into one of four categories according to the communicative content (semantic or emotional) and sensory modality (visual or auditory) of the stimulus. The visual and auditory modalities were chosen because they encompass the bulk of human communication and have been the subject of more research than other sensory modalities. Experiments that could not be classified into these categories were excluded from further analysis. The search returned 499 loci across 239 experiments from an initial pool of 1561 loci across 744 experiments that reported activation within the IFGorb in either hemisphere using any paradigm. Table 1 lists the number of studies and activation loci in each category of experiment.

Table 1

Summary of the number of activation loci and the number of experiments contributing to the kernel density analysis.

\begin{tabular}{llll}
\hline & \multicolumn{2}{l}{ Number of Loci } & \\
\cline { 2 - 3 } & Emotion & Semantics & Sum \\
\hline Audition & 24 & 52 & 76 \\
Vision & 231 & 193 & 424 \\
Sum & 255 & 245 & 499 \\
& Number of Experiments & & \\
\cline { 2 - 3 } & Emotion & Semantics & Sum \\
& 17 & 27 & 44 \\
Audition & 145 & 92 & 237 \\
Vision & 162 & 119 & 281 \\
Sum & & & \multirow{2}{*}{} \\
\hline
\end{tabular}



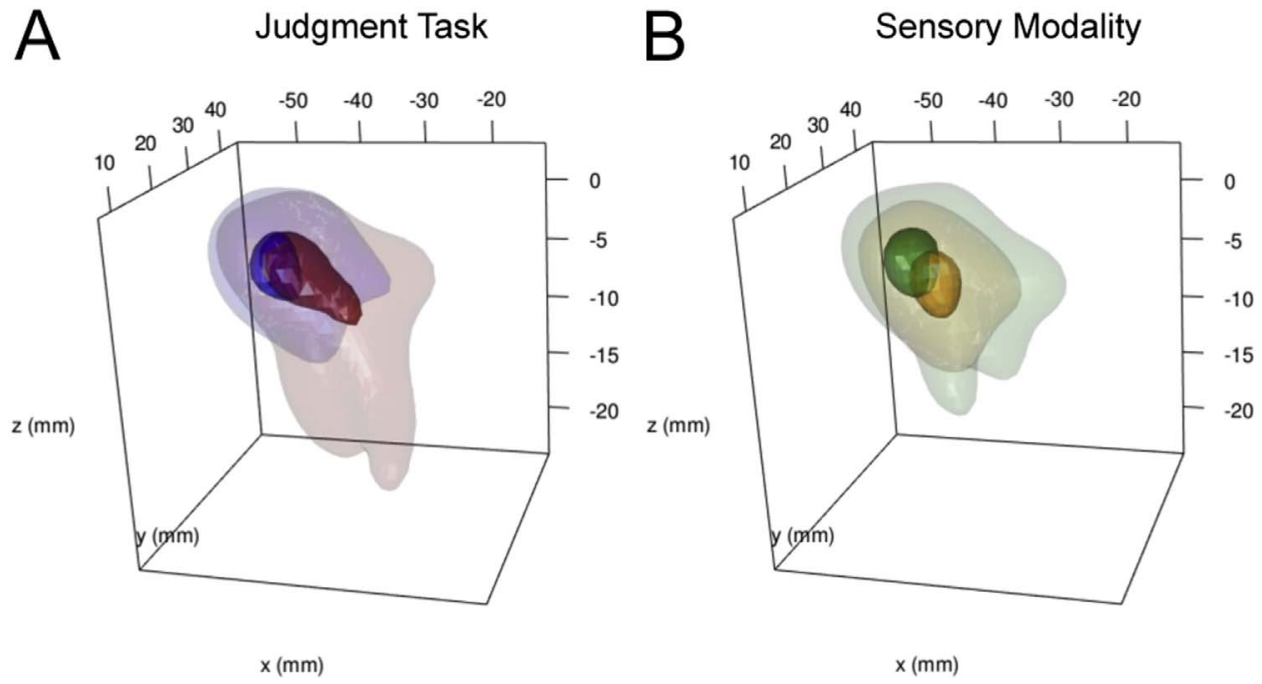

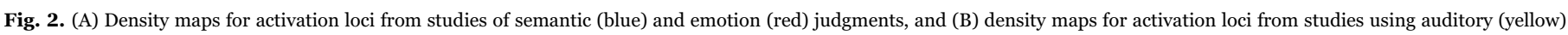

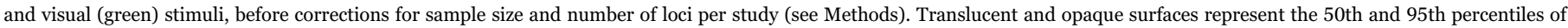
density, respectively.

\section{Analysis}

We used KDE, as implemented in the 'ks' package in R (v3.1.1), to model the distribution of activation peaks within the IFGorb using a Gaussian kernel with empirically determined bandwidths (Duong, 2013, 2015; R Core Team, 2014; Wand and Jones, 1994). KDE creates a smooth estimate of the density, or local abundance, of points within a common space. Although we are not aware of previous brain imaging studies having used this approach, it is a common approach to spatial data in other scientific disciplines. For example, KDE has been used to map hot-spots for traffic accidents in major cities (Harirforoush and Bellalite, 2016), to identify vulnerable habitats (Kenchington et al., 2014), and to model the epidemiology of infectious diseases (Shiode et al., 2015). Belyk et al. (submitted) demonstrate the use of KDE for cognitive neuroscience.

When applied to the peak activations reported in brain imaging studies, KDE provides a smooth representation of the local abundance of peak activations across stereotaxic space. This approach is conceptually similar to Kernel Density Analysis (KDA) and to the more commonly used Activation Likelihood Estimation (ALE), which also create smooth estimates of the local abundance of peak activations (Eickhoff et al., 2012; Turkeltaub et al., 2002; Wager et al., 2007). Like the other approaches, KDE performs hypothesis testing by comparing observed local densities (or likelihoods) to an empirical null distribution estimated by Monte-Carlo simulation. ALE and KDA perform simulations by redistributing the observed activations to random grey matter voxels throughout the brain. The comparison of the observed likelihoods/densities to this random distribution of loci is a valid test of the convergence of activation loci only if the entire brain volume is sampled in the studies contributing to the analysis. This assumption is not met by the present data since it is restricted to a single brain region. We instead performed permutation tests by randomly assigning observed activation loci to conditions, which makes no assumption about the extent of brain coverage.

Four density maps were generated to represent the relative density of activation loci across the IFGorb for studies of i) semantic judgments or ii) emotion judgments, and of iii) auditory stimuli or iv) visual stimuli. Density maps were compared using voxel-wise permutation tests at an isotropic resolution of $1 \mathrm{~mm}^{3}$. First, an observed density difference map was calculated between pairs of conditions. We then estimated an empirical null distribution of local differences in activation loci density by randomly assigning observations to conditions and re-estimating density difference maps across 1000 permutations. The observed activation density difference maps were compared to an empirical null distribution with a threshold of $\mathrm{p}<0.01$. Voxel-wise multiple-comparisons corrections were not applied because these methods may not be appropriate when making topological inferences from spatially continuous data (Chumbley and Friston, 2009) and since the number of possible topological features within the study region was relatively small. To avoid making inferences about regions of stereotaxic space that contained few observations, analyses were constrained to volumes where activation loci were found in high density by masking statistical parametric maps to voxels falling within the 95th percentile of density for either map in a pairwise comparison. This volume was selected as a mask because it aligned well with the anatomical boundaries of the IFGorb and contained the majority of observed loci.

For each pairwise comparison, the condition with the larger sample size was down-sampled to match the sample size of the smaller condition in order to avoid biasing density estimates towards the larger sample. Down-sampling was accomplished by estimating the density of a random sample of observations from the larger set of loci, and taking the mean of the estimates across 1000 iterations. To avoid biasing estimates towards studies that reported multiple loci of activation within the study region, the contribution of each point was weighted by the inverse of the number of loci reported within the IFGorb by its experiment of origin. Hence, each experiment in the dataset contributed equally to the density maps. Images are displayed on a Talairachnormalized template brain using Mango v3.4 (ric.uthscsa.edu/mango).

\section{Results}

We observed that the bulk of the activation loci in this dataset was found in the lateral and superficial portion of the IFGorb adjacent to the IFG pars triangularis, which we will refer to as the lateral zone (centroid: -43, 26, -4). However, while activations from studies of semantic content perception were primarily restricted to this lateral zone, activation loci from studies of emotion perception also extended deep within the frontal operculum adjacent to the ventral anterior insula, which we will refer to as the opercular zone (centroid: $-33,21$, -11). Fig. 2 plots density distributions by both judgment task and sensory modality in stereotaxic space.

Direct tests of activation-loci density revealed a significantly greater density for studies of semantic-content perception in the lateral zone, reflecting the greater concentration of these studies compared to the more widely distributed studies of emotional content perception. In contrast, there was a greater density of activation loci for studies of emotional content processing in the opercular zone, since few studies 
Table 2

Coordinates of cluster centroids from permutation tests on the density distributions of loci of activation from brain imaging studies of semantic-content perception and emotion perception. There was a local increase in the density of loci related to semantic-content perception in the lateral IFGorb, where these loci were found almost exclusively. There was a local increase in the density of loci related to emotion perception in the opercular IFGorb, where semantic-content experiments rarely reported activation loci.

\begin{tabular}{llllll}
\hline \multicolumn{2}{l}{ Semantic $>$ Emotion } & & & & \\
\hline Zone & Size $\left(\mathrm{mm}^{3}\right)$ & $\mathrm{z}$-score & $\mathrm{x}$ & $\mathrm{y}$ & $\mathrm{z}$ \\
\hline Lateral & 351 & 3.74 & -40 & 28 & -9 \\
Lateral & 140 & 3.17 & -50 & 33 & -4 \\
Lateral & 18 & 3.17 & -32 & 18 & -4 \\
Lateral & 5 & 2.44 & -49 & 25 & 0 \\
& & & & & \\
Emotion & \multicolumn{1}{l}{ Semantic } & & & & \\
\hline Zone & Size $\left(\mathrm{mm}^{3}\right)$ & $\mathrm{z}$-score & $\mathrm{x}$ & $\mathrm{y}$ & $\mathrm{z}$ \\
\hline Opercular & 320 & 3.54 & -24 & 20 & -14 \\
Opercular & 222 & 4.07 & -39 & 19 & -16 \\
Opercular & 65 & 3.72 & -25 & 30 & -4 \\
Opercular & 9 & 2.59 & -35 & 31 & -7 \\
\hline
\end{tabular}

of semantics reported activation loci in this zone (see Table 2). Fig. 3 maps density distributions and parametric tests onto a standard neuroanatomical image. Regarding sensory modality, no differences were observed between studies using auditory and visual stimuli. The density distributions of semantic and emotion loci within each sensory modality were similar to the overall trend (see Supplementary Figure S1).

\section{Meta-Analysis 2}

Methods

We performed a second set of meta-analyses to explore the networks of brain regions that co-activate with the lateral and opercular zones of the IFGorb using the method of Meta-Analytic Connectivity Modeling (MACM), as described by Eickhoff et al. (2011). We searched the BrainMap database using Sleuth software v2.4 on March $21^{\text {st }} 2016$ for experiments reporting loci of activation i) within the lateral zone of the IFGorb or ii) within the opercular zone of the IFGorb. This search was conducted using region-of-interest masks of Emotion AND Semantics (identifying the lateral zone) and Emotion NOT Semantics (identifying the opercular zone). Separate metaanalyses were conducted for the lateral and opercular zones using ALE (Eickhoff et al., 2009, 2012; Turkeltaub et al., 2002), as implemented in GingerALE (v2.3.6). Experiments were grouped according to participant pools, as suggested by Turkeltaub et al. (2012). These searches yielded 6850 loci across 417 experiments for the lateral zone, and 2579 loci across 168 experiments for the opercular zone. The conjunction of co-activation network maps for the lateral zone and opercular zone, as well as contrasts between these maps, were assessed with a cluster-wise threshold of $\mathrm{p}<0.05$ from 1000 permutations and a cluster-forming threshold of $\mathrm{p}<0.001$, following the recommendations of Eickhoff et al. (2016).

\section{Results}

Both the lateral zone and opercular zone were consistently coactivated with a sensorimotor network (see Fig. 4 and Table 3), including the bilateral inferior frontal gyrus (BA 47/45/44) extending into the precentral gyrus (BA 6), thalamus, ipsilateral superior temporal sulcus (BA 22), fusiform gyrus (BA 37), inferior parietal lobule (BA 39), anterior cingulate cortex (BA 32), medial frontal cortex (BA 9), and contralateral putamen. Both zones co-activated with the


Fig. 3. (A) The 95th percentile of density maps for studies of the processing of the semantic (blue) or emotional (red) content of communicative signals after carrying out corrections for sample size and number of loci reported per study, and as mapped onto a template brain in Talairach space. (B) Parametric map of the statistical contrast between emotion and semantic density maps. A volume deep within the ventral frontal operculum (red) was more densely populated by loci from studies of emotion judgments, since few studies of semantic processing were observed in this zone. A volume on the lateral surface (blue) was more densely populated by loci from studies of semantic processing. However, we note that the lateral zone was not exclusive to loci from semantic studies and that there was also a high density of loci from emotion studies. Rather, studies of semantic processing only engaged the lateral zone, resulting in a greater density of semantic loci in this zone.

ipsilateral amygdala. The lateral zone further co-activated with a prefrontal network, including the middle frontal gyrus (BA 9), dorsal anterior cingulate cortex (BA 32), and supplementary motor area (BA 6). Separate analyses of experiments that used auditory or visual stimuli had broadly similar results, with differences primarily in auditory and visual association areas (see Figure S2, Supplementary Tables 1 and 2). These coactivation profiles should not be confused with "functional connectivity". They only reflect a set of brain areas that respond to similar experimental manipulations, not temporally-correlated brain areas, as required by the formal definition of functional connectivity (Friston, 1994, 2011). 



Mid. FG

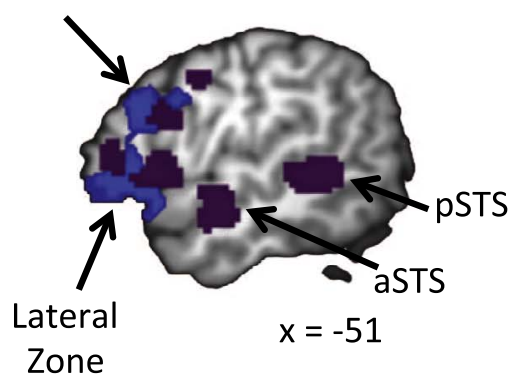

IFG/PrCG

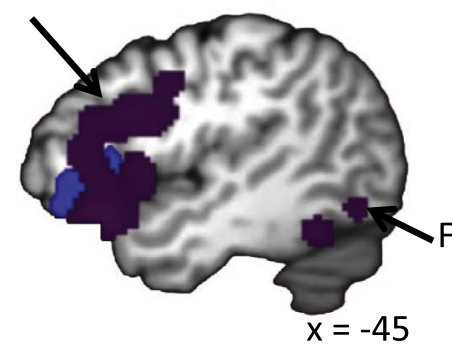

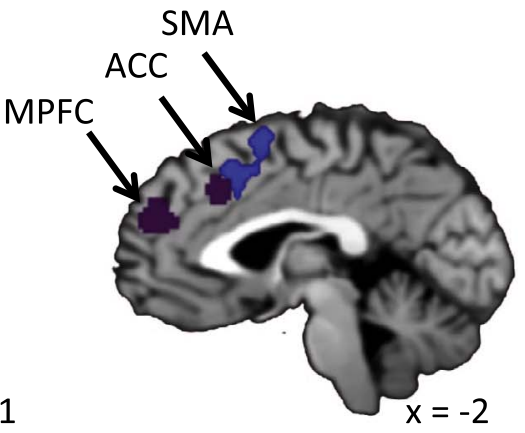

IFG/PrCG


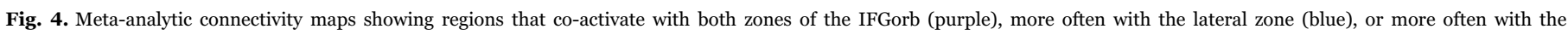

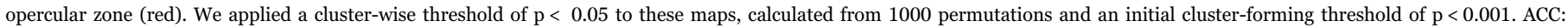

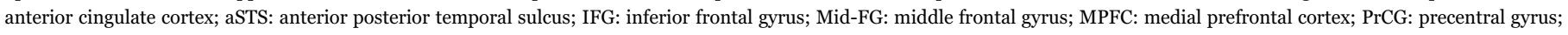
pSTS: posterior superior temporal sulcus; SMA: supplementary motor area.

Table 3

Coordinates of cluster centroids for meta-analytic connectivity maps for the conjunction [Lateral Zone $\cap$ Opercular Zone], and the contrasts [Lateral Zone > Opercular Zone] and [Opercular Zone $>$ Lateral Zone]. We applied a cluster-wise threshold of $\mathrm{p}<0.05$ to these maps, calculated from 1000 permutations of an initial cluster-forming threshold of $\mathrm{p}<0.001$. ACC: anterior cingulate cortex; aSTS: anterior superior temporal sulcus; IFG: inferior frontal gyrus; IPL: inferior parietal lobule; Mid-FG: middle frontal gyrus; MPFC: medial prefrontal cortex; PrCG: precentral gyrus; pSTS: posterior superior temporal sulcus; SMA: supplementary motor area.

\begin{tabular}{lllll}
\hline Conjunction & & & & \\
\hline Region & $\mathrm{x}$ & $\mathrm{y}$ & $\mathrm{z}$ & Size $\left(\mathrm{mm}^{3}\right)$ \\
\hline IFG/PrCG (BA 47/45/44/6) & -38 & 21 & 0 & 22567 \\
ACC (BA 32) & -6 & 24 & 41 & 2379 \\
MPFC (BA 9) & -4 & 50 & 26 & 2245 \\
pSTS (BA 22) & -55 & -40 & 2 & 3049 \\
aSTS (BA 22) & -52 & -7 & -8 & 1619 \\
Fusiform gyrus (BA 37) & -41 & -58 & -14 & 2887 \\
IPL (BA 39) & -40 & -63 & 33 & 135 \\
Amygdala & -20 & -3 & -11 & 2677 \\
Thalamus & -11 & -10 & 6 & 1239 \\
IFG (BA 47/45/44/6) & 38 & 20 & 4 & 17579 \\
Thalamus & 11 & -6 & 4 & 249 \\
Putamen & 23 & -1 & 3 & 129 \\
& & & & \\
Lateral Zone > Opercular Zone & & & Size (mm \\
\hline Region & $\mathrm{x}$ & $\mathrm{y}$ & $\mathrm{z}$ & Size (mm \\
\hline Lateral Zone (BA 47) & -46 & 24 & 2 & 3324 \\
Mid. FG (BA 9) & -54 & 10 & 24 & 992 \\
SMA (BA 6) & -2 & 13 & 47 & 786 \\
ACC (BA 32) & -6 & 11 & 45 & 280 \\
Opercular Zone $>$ Lateral Zone & & & & \\
\hline Region & -29 & 15 & -14 & 8163 \\
\hline Opercular Zone (BA 47) & & & & \\
\hline & & & & \\
\hline
\end{tabular}

\section{General discussion}

Our analyses revealed two distinct functional zones within the IFGorb. First, a lateral zone, located immediately below the anterior horizontal ramus of the lateral sulcus, contained the highest density of activation loci from experiments that reported activation within the IFGorb across study categories. A second zone, extending from the ventral surface adjacent to the orbital gyrus into the ventral frontal operculum, contained the greatest density of loci from studies in which participants made judgments about the emotional content of communicative signals, such as vocal, facial, gestural, and musical expressions of emotion. The convergence of semantics and emotion in the lateral IFGorb provides evidence for a neural hub that might integrate the semantic and expressive aspects of communication.

Both historical and contemporary research using a range of methodological approaches corroborates this division of the IFGorb into distinct lateral and opercular zones. The parcellation aligns with the hierarchical classification of Vogt (1910) based on myeloarchitecture, in which the ventral frontal operculum (Vogt's areas 63-66) is distinct from the remainder of the IFG (Vogt's areas 57-62). Modern parcellation, based on neuroimaging-based measurements of structural and functional connectivity, has observed a similar division into lateral and opercular zones in both humans and monkeys (Neubert, Mars, Thomas, Sallet, and Rushworth, 2014; Neubert, Mars, Sallet, and Rushworth, 2015). Based on receptor architectonics, Amunts et al. (2010) observed that the human frontal operculum contains two subregions, although these were located more dorsally than the opercular zone and were most strongly related to the IFG pars triangularis and pars opercularis of Broca's area, rather than to the IFGorb. The parcellation of Amunts et al. (2010) did not identify subregions in the IFGorb, suggesting that differences between the lateral and opercular zones are not related to differences in neurotransmitter receptors. A recent atlas of the human brain based on the combination of cortical myelin content, cortical thickness, task-based fMRI, and resting functional connectivity observed still finer parcellation throughout the IFG, consistent with Vogt's early findings (Glasser et al., 2016). 


\section{The lateral zone}

Among studies reporting activations within the IFGorb, the lateral zone of the IFGorb was common to studies in which participants processed either semantic or emotional content across sensory modalities. This finding suggests that the lateral zone is a major point of convergence for the perception of semantic and emotional signals during communication. The multi-functionality of the lateral zone is supported by its tendency to coactivate with both limbic nuclei and the prefrontal cortex. The lateral zone is also multimodal, since it is implicated in communicative functions across modalities, for instance the semantics of both spoken and signed words, and the expression of emotion in the voice, face, and body. This integration is consistent with the coactivation of the IFGorb with both auditory and visual association cortex. These networks match the major anatomical connections of the IFGorb with auditory association cortex via the arcuate fasciculus (Frühholz et al., 2015) and uncinate fasciculus (Kier et al., 2004), with visual cortex via the inferior fronto-occipital fasciculus (Sarubbo et al., 2013), as well as with prefrontal cortex and basal forebrain limbic areas (Anwander et al., 2007; Catani et al., 2012; Yeterian et al., 2012). In addition to receiving projections from unisensory auditory and visual cortices, the IFGorb was strongly connected with the pSTS, which has a very similar response profile to the IFGorb as a multisensory area that combines expressive cues across the voice, face, and body (Biau et al., 2016; Campanella and Belin, 2007; Deen et al., 2015; Kreifelts et al., 2009). This is supported by structural connectivity between the pSTS and IFGorb (Frühholz, et al., 2015), suggesting that the role of the IFGorb may go beyond integrating information across sensory modalities, since some of the inputs that it receives may have been already integrated in other brain areas.

The position of the lateral zone between networks for semantic and emotion perception, the input it receives from auditory and visual pathways, as well as its connectivity with both the semantic network and limbic system (Neubert et al., 2014) all suggest that it may aggregate information across multiple channels of communication. This integration may support the comprehension of not only literal linguistic meanings - where semantic and emotional cues support a common message - but also non-literal meanings, such as sarcasm, hyperbole, euphemism, banter, metaphor, and irony, where the information gleaned by combining semantic and emotional cues may be greater than the sum of their parts. Indeed, a meta-analysis of brain imaging studies of non-literal language comprehension observed the strongest association with a cluster centered in the left anterior horizontal ramus of the lateral sulcus (Rapp et al., 2012), which is the macroanatomical landmark that separates the lateral IFGorb from Broca's area.

Emotional vocalizations are auditory objects just as emotional faces and postures are instances of visual objects (Schirmer and Kotz, 2006). These objects are processed with increasing integration and complexity as they progress through their respective sensory systems. The IFGorb appears to be an early point of contact of higher-order auditory and visual association areas with the prefrontal cortex. The convergence of multiple channels of emotional and semantic communication in the lateral zone suggests that it may combine information across modalities to form an integrated evaluation of the message being communicated. Coactivation of the lateral zone and prefrontal cortical areas, such as the middle frontal gyrus, ACC, and SMA, that are involved in outcome monitoring and goal-directed action selection (Ridderinkhof et al., 2004), suggests that the lateral zone may inform the selection of behavioral responses to an individual's social environment.

\section{The opercular zone}

The opercular zone had a clear specialization for processing signals that communicate emotion, and its coactivation profile was more strongly weighted towards the limbic system. Rather than conveying information, the goal of emotional communication may be to influence the behavior of conspecifics, possibly by evoking automatic physiological responses in the perceiver (Owren and Rendall, 2001). For example, the high amplitude and sharp onset of distress calls makes them well suited to the purpose of rapidly attracting the attention of potential allies. This view is supported by the emergence of "structuralmotivational" rules that govern the form of emotional expressions across species (Morton, 1977; Owings and Morton, 1998). One possibility is that the opercular zone may mediate affective and physiological responses to perceiving the emotions of others. Previous studies have observed correlations between activation in the opercular zone and autonomic responses in the peripheral nervous system (Nugent et al., 2011; Park and Thayer, 2014). However, the observation that the IFGorb is more strongly engaged during explicit recognition tasks than during passive perception is difficult to reconcile with the automatic nature of autonomic responses (Witteman et al., 2011). An alternative possibility is that the opercular zone may probe the limbic system for information on an individual's current affective state, which may influence the perception of the emotions of others visa-vis embodied cognition (Neal and Chartrand, 2011; Niedenthal, 2007). Neubert et al. (2014, 2015) observed that, whereas the strongest prefrontal connections of the lateral zone are with the IFG pars triangularis, IFG pars opercularis, and middle frontal gyrus, the strongest prefrontal connections of the opercular zone are with the amygdalae and medial prefrontal cortex. The latter area is a key node in the mentalizing network (Schurz et al., 2014). Our MACM analysis supports a strong connection between the opercular zone and the medial prefrontal cortex. This pattern of coactivation indicates that, unlike the lateral zone, whose coactivation profile is strongly suggestive of an involvement in speech and language, the opercular zone may have a greater involvement in social cognition. Indeed, machine-learning algorithms can decode emotional states from patterns of activation in the opercular zone, suggesting that this area may track the emotional states of others (Kotz et al., 2013).

\section{Lateralization and lack thereof}

The classical neurological perspective attributes propositional language to the left cerebral hemisphere and the expression of emotions to the right cerebral hemisphere. This position was founded, in part, on the views of Hughlings-Jackson $(1878,1879)$, who observed in his medical practice that aphasia, resulting from trauma to the left cerebral hemisphere, primarily affected propositional speech, but could spare certain automatic speech patterns, such as interjections in emotional contexts. Neurological studies in the $20^{\text {th }}$ century disproportionately sampled patients with right hemisphere lesions, leading to the belief that impairments of emotional communication were a syndrome of the right hemisphere, parallel to aphasia as a syndrome of the left hemisphere (Gorelick and Ross, 1987; Ross and Mesulam, 1979). However, meta-analysis and broader sampling practices later demonstrated that, while the effect of right hemisphere damage results in marginally more severe deficits in emotional expression, such deficits are as likely to result from damage to either hemisphere (Witteman et al., 2011). This bilaterality is also supported by neuroimaging studies of both facial and vocal emotional expression (Belyk and Brown, 2014; Fusar-Poli et al., 2009a, 2009b; Witteman et al., 2012).

Our meta-analyses revealed strong left lateralization of IFGorb activation in the neuroimaging literature on the perception of semantic content, but a lack of lateralization in the neuroimaging literature on emotion perception across communicative domains. This follows from an emerging perspective that emotional expression across domains may be processed by a common bilateral network (Brück et al., 2011; Frühholz et al., 2016). 


\section{An evolutionary paradox}

Given that non-human primates readily employ emotional expressions but are relatively poor in symbolic language use (Arbib et al., 2008), how did the lateral IFGorb come to acquire semantic functionality from an ancestral area whose function was likely emotional? The IFGorb (BA 47) in humans corresponds to Walker's area 12 in macaques (Macaca mulatta; Petrides and Pandya, 2002). Importantly, this area's parcellation into lateral and opercular zones appears to be shared between species. In humans, the functional connectivity profile of a region approximating the lateral zone is most similar to the macaque area $47 / 12$, while that of a region approximating the opercular zone is most similar to the functional connectivity of the macaque's lateral agranular insula (Neubert et al., 2014, 2015). The relatively large phylogenetic distance between humans and macaques within the primate order suggests that this correspondence may hold across primates and may therefore be representative of a common primate ancestor.

Although great apes can identify objects and their likely uses, their ability to use symbols in place of objects is limited to approximately the level of human children (Lyn et al., 2011). With extensive training, chimpanzees (Pan troglodytes) and gorillas (Gorilla gorilla) can learn dozens or hundreds of vocabulary items in American Sign Language (Gardner and Gardner, 1985; Patterson and Cohn, 1990). In addition, there is some evidence that a minimal form of these behaviors occurs in the wild in some species (Arbib et al., 2008). While such findings do demonstrate some capacity for semantic communication in great apes, their abilities fall short of the extent of the human vocabulary and the ease with which it is acquired.

We are not aware of similar studies conducted with the species of primates for which comparative neuroscience data are available (such as macaques), although it may be presumed that the semantic abilities of these species are even more limited. Hence, the IFGorb appears to have evolved in lower primates even in the absence of a strong capacity for referential semantics with which it is associated in humans. The preserved morphology, but marked change in function, of the IFGorb across the primate order therefore presents somewhat of a paradox.

One possible solution is that the human IFGorb may have acquired new connections as part of the evolution of the semantic network. The human and monkey lateral zones share extensive frontal and temporal connectivity (Neubert et al., 2014). Hence, many of the connections between the IFGorb and brain areas that would become important to other aspects of language, such as the IFG pars triangularis (BA 45) and IFG pars opercularis (BA 44) that are implicated in syntactic and phonological processes, may have been in place prior to the evolution of language. We hypothesize that the human lateral IFGorb may have formed novel connections with more distal brain regions, allowing it to inerface with the emerging semantic network. A candidate for such a target of connectivity is the angular gyrus (Neubert et al., 2014), which in humans is involved in both semantics and social cognition (Binder and Desai, 2011; Schurz et al., 2014). The IFGorb may have initially evolved to support the comprehension of emotional signals, being later co-opted to support semantic communication in humans by forming new connections with brain regions that formed the human semantic network.

\section{Conclusion}

The IFGorb contains at least two functionally specialized divisions that we have referred to as the lateral and opercular zones. The lateral zone may be a locus of convergence between semantic and emotional channels of communication, while the opercular zone may be more specialized for understanding the social meanings of the emotional states communicated by others. This finding has implications for the further study of both semantic and emotional-expression processing, as both fields have independently attributed domain-specific functions to the IFGorb. The convergence of semantics and emotional expression in the lateral zone suggests that these functions may be more meaningfully studied together than apart. The IFGorb may provide a mechanism by which channels of communication are integrated to support the interpretation of statements with the rich nuances of meaning typical of human communication, including sarcasm, hyperbole, euphemism, banter, metaphor, and irony.

\section{Acknowledgments}

This work was funded by grants from the Auditory Cognitive Neuroscience Society (ACN) to MB and SAK, the Natural Sciences and Engineering Research Council (NSERC) of Canada to SB (0468615), and the Biotechnology and Biological Science Research Council (BBSRC) of the UK to SAK (BB/M009742/1).

\section{Appendix A. Supporting information}

Supplementary data associated with this article can be found in the online version at http://dx.doi.org/10.1016/j.neuroimage.2017.04. 020 .

\section{References}

Arbib, M.A., Liebal, K., Pika, S., 2008. Primate vocalization, gesture, and the evolution of human language. Curr. Anthropol. 49, 1053-1076.

Amunts, K., Lenzen, M., Friederici, A.D., Schleicher, A., Morosan, P., PalomeroGallagher, N., Zilles, K., 2010. Broca's region: novel organizational principles and multiple receptor mapping. PLoS Biol. 8, 1-16. http://dx.doi.org/10.1371/ journal.pbio.1000489.

Anwander, A., Tittgemeyer, M., von Cramon, D.Y., Friederici, A.D., Knösche, T.R., 2007. Connectivity-based parcellation of Broca's area. Cereb. Cortex 17, 816-825. http:// dx.doi.org/10.1093/cercor/bhk034.

Belyk, M., Brown, S., 2014. Perception of affective and linguistic prosody: an ALE metaanalysis of neuroimaging studies. Soc. Cogn. Affect. Neurosci. 9, 1395-1403. http:// dx.doi.org/10.1093/scan/nst124.

Belyk, M., Brown, S., 2016. Pitch underlies activation of the vocal system during affective vocalization. Soc. Cogn. Affect. Neurosci. 11, 1078-1088. http://dx.doi.org/ $10.1093 / \mathrm{scan} / \mathrm{nsv} 074$.

Belyk, M., Brown, S., Kotz, S.A., (submitted). Demonstration and validation of kernel density estimation for spatial meta-analyses of neuroimaging data. Data in Brief.

Biau, E., Morís Fernández, L., Holle, H., Avila, C., Soto-Faraco, S., 2016. Hand gestures as visual prosody: bold responses to audio-visual alignment are modulated by the communicative nature of the stimuli. Neuroimage 132, 129-137. http://dx.doi.org/ 10.1016/j.neuroimage.2016.02.018.

Binder, J.R., Desai, R.H., 2011. The neurobiology of semantic memory. Trends Cogn. Sci. 15, 527-536. http://dx.doi.org/10.1016/j.tics.2011.10.001.

Binder, J.R., Desai, R.H., Graves, W.W., Conant, L., 2009. Where Is the semantic system? A critical review and meta-analysis of 120 functional neuroimaging studies. Cereb. Cortex 19, 2767-2796. http://dx.doi.org/10.1093/cercor/bhp055.

Brodmann, K., 1909. Localisation in the Cerebral Cortex 3rd ed.. Springer, New York.

Brück, C., Kreifelts, B., Wildgruber, D., 2011. Emotional voices in context: a neurobiological model of multimodal affective information processing. Phys. Life Rev. 8, 383-403. http://dx.doi.org/10.1016/j.plrev.2011.10.002.

Campanella, S., Belin, P., 2017. Integrating face and voice in person perception. Trends Cogn. Sci. 11, 535-543. http://dx.doi.org/10.1016/j.tics.2007.10.001.

Catani, M., Dell'acqua, F., Vergani, F., Malik, F., Hodge, H., Roy, P., Valabregue, R., Thiebaut de Schotten, M., 2012. Short frontal lobe connections of the human brain. Cortex 48, 273-291. http://dx.doi.org/10.1016/j.cortex.2011.12.001.

Chumbley, J., Friston, K., 2009. False discovery rate revisited: FDR and topological inference using Gaussian random fields. Neuroimage 44, 62-70. http://dx.doi.org/ 10.1016/j.neuroimage.2008.05.021.

Craddock, R.C., James, G.A., Iii, P.E.H., Hu, X.P., Mayberg, H.S., 2012. A whole brain fMRI atlas generated via spatially constrained spectral clustering. Hum. Brain Mapp 33, 1914-1928. http://dx.doi.org/10.1002/hbm.21333.A.

Deen, B., Koldewyn, K., Kanwisher, N., Saxe, R., 2015. Functional organization of social perception and cognition in the superior temporal sulcus. Cereb. Cortex 25, 4596-4609.

Dietrich, S., Ackermann, H., Szameitat, D.P., Alter, K., 2006. Psychoacoustic studies on the processing of vocal interjections: how to disentangle lexical and prosodic information? Prog. Brain Res. 156, 295-302.

Duong, T., 2013. Local significant differences from nonparametric two-sample tests. J. Nonparametr. Stat. 25, 635-645. http://dx.doi.org/10.1080/ 10485252.2013 .810217$.

Duong, T., 2015. ks: Kernal Smoothing. R Package version 1.9.4.

Eickhoff, S.B., Bzdok, D., Laird, A.R., Kurth, F., Fox, P.T., 2012. Activation likelihood estimation meta-analysis revisited. Neuroimage 59, 2349-2361. http://dx.doi.org/ 10.1016/j.neuroimage.2011.09.017. 
Eickhoff, S.B., Bzdok, D., Laird, A.R., Roski, C., Caspers, S., Zilles, K., Fox, P.T., 2011. Coactivation patterns distinguish cortical modules, their connectivity and functional differentiation. Neuroimage 57, 938-949. http://dx.doi.org/10.1016/ j.neuroimage.2011.05.021.

Eickhoff, S.B., Laird, A.R., Grefkes, C., Wang, L.E., Zilles, K., Fox, P.T., 2009. Coordinatebased activation likelihood estimation meta-analysis of neuroimaging data: a random-effects approach based on empirical estimates of spatial uncertainty. Hum. Brain Mapp. 30, 2907-2926. http://dx.doi.org/10.1002/hbm.20718.

Eickhoff, S.B., Nichols, T.E., Laird, A.R., Hoffstaedter, F., Amunts, K., Fox, P.T., Bzdok, D., Eickhoff, C.R., 2016. Behavior, sensitivity, and power of activation likelihood estimation characterized by massive empirical simulation. Neuroimage 137, 70-85. http://dx.doi.org/10.1016/j.neuroimage.2016.04.072.

Filippi, P., 2016. Emotional and interactional prosody across animal communication systems: a comparative approach to the emergence of language. Front Psychol. 7, 1393.

Friston, K.J., 1994. Functional and effective connectivity: a synthesis. Hum. Brain Mapp. 2, $56-78$.

Friston, K.J., 2011. Functional and effective connectivity: a review. Brain Connect. 1, $13-36$.

Frühholz, S., Gschwind, M., Grandjean, D., 2015. Bilateral dorsal and ventral fiber pathways for the processing of affective prosody identified by probabilistic fiber tracking. Neuroimage 109, 27-34. http://dx.doi.org/10.1016/ j.neuroimage.2015.01.016.

Frühholz, S., Trost, W., Kotz, S.A., 2016. The sound of emotions: towards a unifying neural network perspective of affective sound processing. Neurosci. Biobehav. Rev. 68, 96-110. http://dx.doi.org/10.1016/j.neubiorev.2016.05.002.

Fusar-Poli, P., Placentino, A., Carletti, F., Landi, P., Allen, P., Surguladze, S., Benedetti, F., Abbamonte, M., Gasparotti, R., Barale, F., 2009a. Functional atlas of emotional faces processing: A voxel-based meta-analysis of 105 functional magnetic resonance imaging studies. J. Psychiatry Neurosci. 34, 418-432.

Fusar-Poli, P., Placentino, A., Carletti, F., Allen, P., Landi, P., Abbamonte, M., Barale, F., Perez, J., McGuire, P., Politi, P.L., 2009b. Laterality effect on emotional faces processing: ALE meta-analysis of evidence. Neurosci. Lett. 452, 262-267. http:// dx.doi.org/10.1016/j.neulet.2009.01.065.

Gardner, B.T., Gardner, R.A., 1985. Signs of intelligence in cross-fostered chimpanzees. Philos. Trans. R. Soc. Lond. B. Biol. Sci. 308, 159-176. http://dx.doi.org/10.1098/ rstb.1985.0017.

Glasser, M.F., Coalson, T.S., Robinson, E.C., Hacker, C.D., Harwell, J., Yacoub, E., Ugurbil, K., Andersson, J., Beckmann, C.F., Jenkinson, M., Smith, S.M., Van Essen, D.C., 2016. A multi-modal parcellation of human cerebral cortex. Nature 536, 171-178. http://dx.doi.org/10.1038/nature18933.

Gorelick, P.B., Ross, E.D., 1987. The aprosodias: further functional-anatomical evidence for the organisation of affective language in the right hemisphere. J. Neurol. Neurosurg. Psychiatry 50, 553-560.

Harirforoush, H., Bellalite, L., 2016. A new integrated GIS-based analysis to detect hotspots: a case study of the city of Sherbrooke. Accid. Anal. Prev., 1-13. http:// dx.doi.org/10.1016/j.aap.2016.08.015.

Hughlings-Jackson, J., 1878. On affections of speech from disease of the brain. Brain 1, 304-330.

Hughlings-Jackson, J., 1879. On affections of speech from disease of the brain. Brain 2, 323-356.

Jessen, S., Kotz, S.A., 2015. Affect differentially modulates brain activation in uni- and multisensory body-voice perception. Neuropsychologia 66, 134-143. http:// dx.doi.org/10.1016/j.neuropsychologia.2014.10.038.

Judaš, M., Cepanec, M., 2010. Oskar Vogt: the first myeloarchitectonic map of the human frontal cortex. Transl. Neurosci. 1, 72-94. http://dx.doi.org/10.2478/v10134-0100005-z.

Kenchington, E., Murillo, F.J., Lirette, C., Sacau, M., Koen-Alonso, M., Kenny, A., Ollerhead, N., Wareham, V., Beazley, L., 2014. Kernel density surface modelling as a means to identify significant concentrations of vulnerable marine ecosystem indicators. PLoS ONE 9, e109365. http://dx.doi.org/10.1371/ journal.pone.0109365.

Kier, E.L., Staib, L.H., Davis, L.M., Bronen, R.A., 2004. MR imaging of the temporal stem: anatomic dissection tractography of the uncinate fasciculus, inferior occipitofrontal fasciculus, and Meyer's loop of the optic radiation. Am. J. Neuroradiol. 25, 677-691.

Kotz, S.A., Kalberlah, C., Bahlmann, J., Friederici, A.D., Haynes, J.D., 2013. Predicting vocal emotion expressions from the human brain. Hum. Brain Mapp. 34, 1971-1981 . http://dx.doi.org/10.1002/hbm.22041.

Kreifelts, B., Ethofer, T., Shiozawa, T., Grodd, W., Wildgruber, D., 2009. Cerebral representation of non-verbal emotional perception: fMRI reveals audiovisual integration area between voice- and face-sensitive regions in the superior temporal sulcus. Neuropsychologia 47, 3059-3066. http://dx.doi.org/10.1016/ j.neuropsychologia.2009.07.001.

Lancaster, J.L., Rainey, L.H., Summerlin, J.L., Freitas, C.S., Fox, P.T., Evans, A.C., Toga, A.W., Mazziotta, J.C., 1997. Automated labeling of the human brain: a preliminary report on the development and evaluation of a forward-transform method. Hum. Brain Mapp. 5 (4), 238-242 http://dx.doi.org/10.1002/(SICI)1097-0193(1997) 5:43.0.CO;2-4.

Lancaster, J.L., Woldorff, M.G., Parsons, L.M., Liotti, M., Freitas, C.S., Rainey, L., Kochunov, P.V., Nickerson, D., Mikiten, S.A., Fox, P.T., 2000. Automated Talairach Atlas labels for functional brain mapping. Hum. Brain Mapp. 10 (3), 120-131 http://dx.doi.org/10.1002/1097-0193(200007)10:33.0.CO;2-8.

Lehne, M., Rohrmeier, M., Koelsch, S., 2014. Tension-related activity in the orbitofrontal cortex and amygdala: an fMRI study with music. Soc. Cogn. Affect. Neurosci. 9, 1515-1523. http://dx.doi.org/10.1093/scan/nst141.
Levitin, D., Menon, V., 2003. Musical structure is processed in "language" areas of the brain: a possible role for Brodmann Area 47 in temporal coherence. Neuroimage 20, 2142-2152. http://dx.doi.org/10.1016/j.neuroimage.2003.08.016.

Lotze, M., Heymans, U., Birbaumer, N., Veit, R., Erb, M., Flor, H., Halsband, U., 2006 Differential cerebral activation during observation of expressive gestures and motor acts. Neuropsychologia 44, 1787-1795. http://dx.doi.org/10.1016/ j.neuropsychologia.2006.03.016.

Lyn, H., Greenfield, P.M., Savage-Rumbaugh, S., Gillespie-Lynch, K., Hopkins, W.D., 2011. Nonhuman primates do declare! A comparison of declarative symbol and gesture use in two children, two bonobos, and a chimpanzee. Lang. Commun. 31, 63-74. http://dx.doi.org/10.1016/j.langcom.2010.11.001.

MacSweeney, M., Woll, B., Campbell, R., McGuire, P.K., David, A.S., Williams, S.C.R., Suckling, J., Calvert, G.A., Brammer, M.J., 2002. Neural systems underlying British Sign Language and audio-visual English processing in native users. Brain 125, $1583-1593$.

Morton, E., 1977. On the occurrence and significance of motivation-structural rules in some bird and mammal sounds. Am. Nat. 111, 855-869.

Neal, D.T., Chartrand, T.L., 2011. Embodied emotion perception: amplifying and dampening facial feedback modulates emotion perception accuracy. Soc. Psychol. Personal. Sci. 2, 673-678. http://dx.doi.org/10.1177/1948550611406138.

Neubert, F.-X., Mars, R.B., Sallet, J., Rushworth, M.F.S., 2015. Connectivity reveals relationship of brain areas for reward-guided learning and decision making in human and monkey frontal cortex. Proc. Natl. Acad. Sci. USA 11, E2695-E2704. http://dx.doi.org/10.1073/pnas.1410767112.

Neubert, F.X., Mars, R.B., Thomas, A.G., Sallet, J., Rushworth, M.F.S., 2014 Comparison of human ventral frontal cortex areas for cognitive control and language with areas in monkey frontal cortex. Neuron 81, 700-713. http://dx.doi.org/ 10.1016/j.neuron.2013.11.012.

Niedenthal, P.M., 2007. Embodying emotion. Science 316, 1002-1005. http:// dx.doi.org/10.1126/science.1136930.

Nugent, A.C., Bain, E.E., Thayer, J.F., Sollers, J.J., Drevets, W.C., 2011. Heart rate variability during motor and cognitive tasks in females with major depressive disorder. Psychiatry Res. Neuroimaging 191, 1-8. http://dx.doi.org/10.1016/ j.pscychresns.2010.08.013.

Owings, D.H., Morton, E.S., 1998. Animal Vocal Communication: A New Approach.. Cambridge University Press, New York.

Owren, M.J., Rendall, D., 2001. Sound on the rebound: bringing form and function back to the forefront in understanding nonhuman primate vocal signaling. Evol. Anthropol. 10, 58-71. http://dx.doi.org/10.1002/evan.1014.

Park, G., Thayer, J.F., 2014. From the heart to the mind: cardiac vagal tone modulates top-down and bottom-up visual perception and attention to emotional stimuli. Front. Psychol. 5, 1-8. http://dx.doi.org/10.3389/fpsyg.2014.00278.

Patterson, F.G.P., Cohn, R.H., 1990. Language acquisition by a Lowland Gorilla: Koko's first ten years of vocabulary development. Word 7956, 97-143. http://dx.doi.org/ 10.1080/00437956.1990.11435816.

Petrides, M., Pandya, D.N., 2002. Comparative cytoarchitectonic analysis of the human and the macaque ventrolateral prefrontal cortex and corticocortical connection patterns in the monkey. Eur. J. Neurosci. 16, 291-310. http://dx.doi.org/10.1046/ j.1460-9568.2002.02090.x.

Rapp, A.M., Mutschler, D.E., Erb, M., 2012. Where in the brain is nonliteral language? A coordinate-based meta-analysis of functional magnetic resonance imaging studies. Neuroimage 63, 600-610. http://dx.doi.org/10.1016/j.neuroimage.2012.06.022.

R Core Team, 2014. R: a language and environment for statistical computing. R Found. Stat. Comput., Vienna, Austria.〈https://www.R-project.org/〉.

Ridderinkhof, K.R., Wery, P.M., Van Den, Wildenberg W.P.M, Segalowitz, S.J., Carter, C.S., 2004. Neurocognitive mechanisms of cognitive control: the role of prefrontal cortex in action selection, response inhibition, performance monitoring, and rewardbased learning. Brain Cogn. 56, 129-140. http://dx.doi.org/10.1016/ j.bandc.2004.09.016.

Rodd, J.M., Vitello, S., Woollams, A.M., Adank, P., 2015. Localising semantic and syntactic processing in spoken and written language comprehension: an activation likelihood estimation meta-analysis. Brain Lang. 141, 89-102. http://dx.doi.org/ 10.1016/j.bandl.2014.11.012.

Ross, E.D., Mesulam, M., 1979. Dominant language functions of the prosody and emotional gesturing right hemisphere? Arch. Neurol. 36, 144-148.

Ross, E.D., Monnot, M., 2008. Neurology of affective prosody and its functional-anatomic organization in right hemisphere. Brain Lang. 104, 51-74.

Sarubbo, S., De Benedictis, A., Maldonado, I.L., Basso, G., Duffau, H., 2013. Frontal terminations for the inferior fronto-occipital fascicle: anatomical dissection, DTI study and functional considerations on a multi-component bundle. Brain Struct. Funct. 218, 21-37. http://dx.doi.org/10.1007/s00429-011-0372-3.

Scherer, K.R., Ellgring, H., 2007. Multimodal expression of emotion: affect programs or componential appraisal patterns? Emotion 7, 158-171.

Schirmer, A., Kotz, S.A., 2006. Beyond the right hemisphere: brain mechanisms mediating vocal emotional processing. Trends Cogn. Sci. 10, 24-30. http:// dx.doi.org/10.1016/j.tics.2005.11.009.

Schurz, M., Radua, J., Aichhorn, M., Richlan, F., Perner, J., 2014. Fractionating theory of mind: a meta-analysis of functional brain imaging studies. Neurosci. Biobehav. Rev. 42, 9-34. http://dx.doi.org/10.1016/j.neubiorev.2014.01.009.

Shiode, N., Shiode, S., Rod-Thatcher, E., Rana, S., Vinten-Johansen, P., 2015. The mortality rates and the space-time patterns of John Snow's cholera epidemic map. Int. J. Health Geogr. 14, 21. http://dx.doi.org/10.1186/s12942-015-0011-y.

Tabei, K., 2015. Inferior frontal gyrus activation underlies the perception of emotions, while precuneus activation underlies the feeling of emotions during music listening. Behav. Neurol.. http://dx.doi.org/10.1155/2015/529043.

Talairach, J., Tournoux, P., 1988. Co-planar stereotaxic atlas of the human brain. 3- 
Dimensional Proportional System: An Approach to Cerebral Imaging. Georg Thieme Verlag, New York.

Turkeltaub, P.E., Eden, G.F., Jones, K.M., Zeffiro, T.A., 2002. Meta-analysis of the functional neuroanatomy of single-word reading: method and validation. Neuroimage 16, 765-780. http://dx.doi.org/10.1006/nimg.2002.1131.

Turkeltaub, P.E., Eickhoff, S.B., Laird, A.R., Fox, M., Wiener, M., Fox, P., 2012. Minimizing within-experiment and within-group effects in activation likelihood estimation meta-analyses. Hum. Brain Mapp. 33, 1-13. http://dx.doi.org/10.1002/ hbm. 21186.

Vogt, O., 1910. Die myeloarchitektonische Felderung des menschlichen Stirnhirns. J. für Psychol. und Neurol. 15, 221-232.

Wager, T.D., Lindquist, M., Kaplan, L., 2007. Meta-analysis of functional neuroimaging data: current and future directions. Soc. Cogn. Affect. Neurosci. 2, 150-158. http:// dx.doi.org/10.1093/scan/nsm015.
Wand, M., Jones, M., 1994. Multivariate plug-in bandwidth selection. Comput. Stat. 9, 97-116.

Witteman, J., Van Heuven, V.J.P., Schiller, N.O., 2012. Hearing feelings: a quantitative meta-analysis on the neuroimaging literature of emotional prosody perception. Neuropsychologia 50, 2752-2763. http://dx.doi.org/10.1016/ j.neuropsychologia.2012.07.026.

Witteman, J., van Ijzendoorn, M.H., van de Velde, D., van Heuven, V.J.J.P., Schiller, N.O., 2011. The nature of hemispheric specialization for linguistic and emotional prosodic perception: a meta-analysis of the lesion literature. Neuropsychologia 49, 3722-3738. http://dx.doi.org/10.1016/j.neuropsychologia.2011.09.028.

Yeterian, E.H., Pandya, D.N., Tomaiuolo, F., Petrides, M., 2012. The cortical connectivity of the prefrontal cortex in the monkey brain. Cortex 48, 58-81. http://dx.doi.org/ 10.1016/j.cortex.2011.03.004. 